\title{
Exploring and Comparing High Stakes Writing Test Prompts Content Structure
}

\author{
Abdolvahab Khademi \\ University of Massachusetts Amherst \\ Paper presented at Graduate Student Research Session, \\ the 77th Annual Meeting of the National Council on Measurement in Education \\ (April 15-19, 2015), Chicago, Illinois, USA.
}

\begin{abstract}
TOEFL and IELTS are two major tests that measure language preparedness of prospective college students. The writing section of these two tests provide a measure of readiness for academic writing. However, to what extent these two tests measure the same contents has not been quantitatively investigated before. In this paper, multidimensional scaling (MDS) is applied to explore the content structure of writing prompts in TOEFL and IELTS examinations.
\end{abstract}

\section{Introduction}

The present study attempts to explore the content dimensionality of writing prompts used in Test of English as a Foreign Language (TOEFL) and the International English Language Testing System (IELTS), developed and administered by ETS and Cambridge ESOL, respectively. Both tests are used to measure academic language proficiency of non-English speaking students who aspire to study in an English-medium university throughout the world, 
particularly in North America, Australia, and Europe. Both tests are accepted by graduate schools with different cut-off scores and/or component requirements.

Investigating the content structure of writing prompts is a test validation process because a test should assess second language abilities independent of general cognitive abilities (Chapel, Enright \& Jamieson, 2010) and other construct-irrelevant factors, such task complexity, task specificity, task familiarity or cultural bias. Validity is defined as, "the degree to which evidence and theory support the interpretations of test scores entailed by proposed uses of tests" (American Educational Research Association, American Psychological Association, \& National Council on Measurement in Education, 1999). Therefore, in assessing the writing proficiency of students, selection of prompts with no or minimum construct-irrelevant factors plays an important role in the interpretation and use of the test scores obtained.

Of different structural methods, Multidimensional Scaling (MDS) holds a popular place because of its ability to use proximity data. The present study uses (replicated) classical multidimensional scaling method, which can reveal and visualize latent structures in a dataset (Borg \& Groenen, 2005). Cox and Cox (2001) define MDS as, "the search for a low dimensional space, usually Euclidean, in which points in the space represent the objects [...], one point representing one object, and such that the distances between the points in the space [...] match, as well as possible, the original dissimilarities $[\ldots] ”($ p. 1).

\section{Method}

The data comprised pair ratings of writing prompts from official TOEFL and IELTS tests released by ETS and Cambridge ESOL. For this study, 40 prompts (20 from each test) were randomly chosen from the officially released pool of topics and paired with each other to form 
780 pairs $^{1}$. Each pair was rated on a 10 -point Likert scale in terms of cognitive similarity of the prompts.

Rating was conducted through online surveys completed by three raters. The raters were non-native speakers and had formal education in language teaching and applied linguistics at master's and doctorate levels with a minimum of 10 years teaching different language skills in Iran and Canada. The raters did not convene because of geographical distance. The raters were duly briefed about the task. In addition, because the task seemed lengthy, it was divided into multiple parts and completed over a period of 10 days (a fourth rater opted out of the study). One rater was female and two others were male. The raters had to read both prompts in a pair and decide if they were very different or very similar in terms of cognitive demand of the tasks (according to the instruction).

The collected data were entered in IBM SPSS v. 19 in the form of three $40 \times 40$ symmetrical lower triangle matrices. Because there are three similarity matrices, the MDS method used is called replicated MDS, or RMDS. Both PROXSCAL and ALSCAL algorithms were used to scale the data. The level of measurement chosen was ordinal with ties broken. When ties are broken, equal proximities are not necessarily mapped into equal distances (Borg et al. 2013). Missing data were coded 999 in the data matrix. Missing data are skipped in MDS. Ordinal MDS can recover latent structures with even 80\% data missing (Spence \& Domoney, 1974). Thus the MDS model chosen is ordinal or nonmetric RMDS. In addition to ALSCAL procedure, PROXSCAL was also used to aid interpretation of the obtained dimensions. In PROXSCAl, the initial configuration was changed from simplex to Torgerson, as recommended by Borg et al. (2013), which helps avoid suboptimal local minima.

\footnotetext{
${ }^{1}$ In this paper, the results of individual tests and their item pairings are presented. The full study includes crosspairings of the prompts from the two tests.
} 
The most common statistical indicators for evaluating model-data fit in MDS are the loss function (Stress) and R-squared (RSQ). Stress or (S-STRESS, in ALSCAL algorithm) is the square root of a normalized residual sum of squares (Kruskal \& Wish, 1978). After finding the possible dimensions, the choice of the number of interpretable dimensions is based on the Stress value. The lower the Stress values, the better the data fit the model at that specific dimension. The S- Stress loss function in ALSCAL is different in that it weights the fit of large distances more heavily than the fit of the smaller distances (Borg et al., 2013). R-squared (RSQ) is another indicator in MDS which is used for the selection of dimensions on the basis of the variance explained at that particular dimension.

\section{Results}

\section{TOEFL Prompts}

In this section, the ALSCAL and PROXSCAL results are presented for TOEFL writing prompts.

Table 1 and Figure 1 show the results of ALSCAL analysis on the ordinal data of the TOEFL writing topics. The common indicators for goodness-of-fit are the loss function SSTRESS and the R squared (RSQ). The lower the S-STRESS is, the better the solution. For RSQ, we're seeking higher values. 
Table 1: S-Stress and RSQ values for TOEFL writing prompts from ALSCAL analysis.

\begin{tabular}{|l|l|l|l|l|l|l|l|l|}
\hline \multicolumn{9}{|c|}{ TOEFL Writing Prompts } \\
\hline \multirow{2}{*}{ Dim. } & \multicolumn{2}{|c|}{ Averaged } & \multicolumn{2}{c|}{ Matrix 1 } & \multicolumn{2}{c|}{ Matrix 2 } & \multicolumn{2}{c|}{ Matrix 3 } \\
\cline { 2 - 9 } & S-Stress & RSq & S-Stress & RSq & S-Stress & RSq & S-Stress & RSq \\
\hline 6 & .09 & .81 & .09 & .78 & .08 & .84 & .09 & .80 \\
\hline 5 & .10 & .78 & .12 & .72 & .09 & .84 & .10 & .78 \\
\hline 4 & .13 & .74 & .16 & .61 & .10 & .86 & .13 & .77 \\
\hline 3 & .17 & .71 & .21 & .58 & .14 & .82 & .17 & .73 \\
\hline 2 & .24 & .68 & .27 & .58 & .18 & .82 & .25 & .65 \\
\hline 1 & .33 & .68 & .40 & .52 & .24 & .85 & .33 & .68 \\
\hline
\end{tabular}

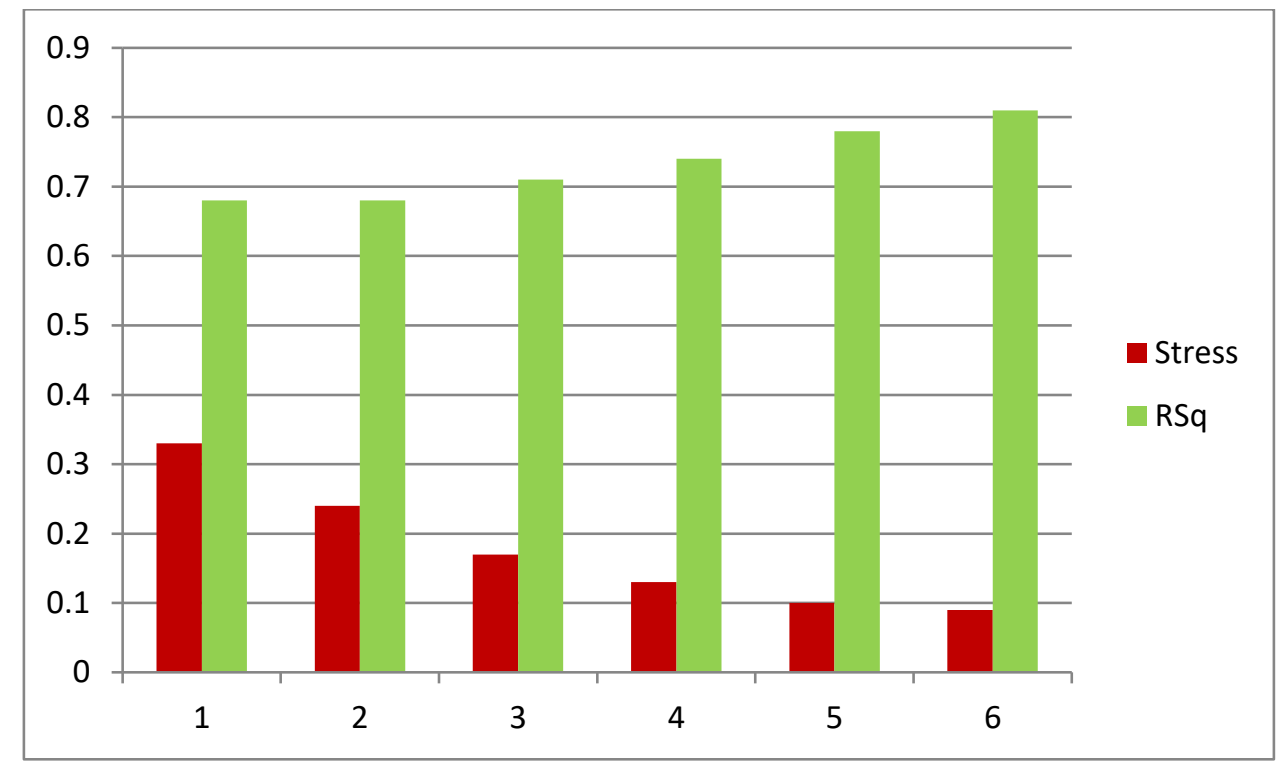

Figure 1: SStress and RSq values at different dimensions of TOEFL Writing Prompts.

As the loss function values show, the Stress improves noticeably going from two dimensions to three dimensions, after which the dip is gradual. Increment in RSQ is almost equal from one dimension to the next higher. Therefore, the ALSCAL results suggest a two- to threedimensional structure to the writing prompts of TOEFL test. 


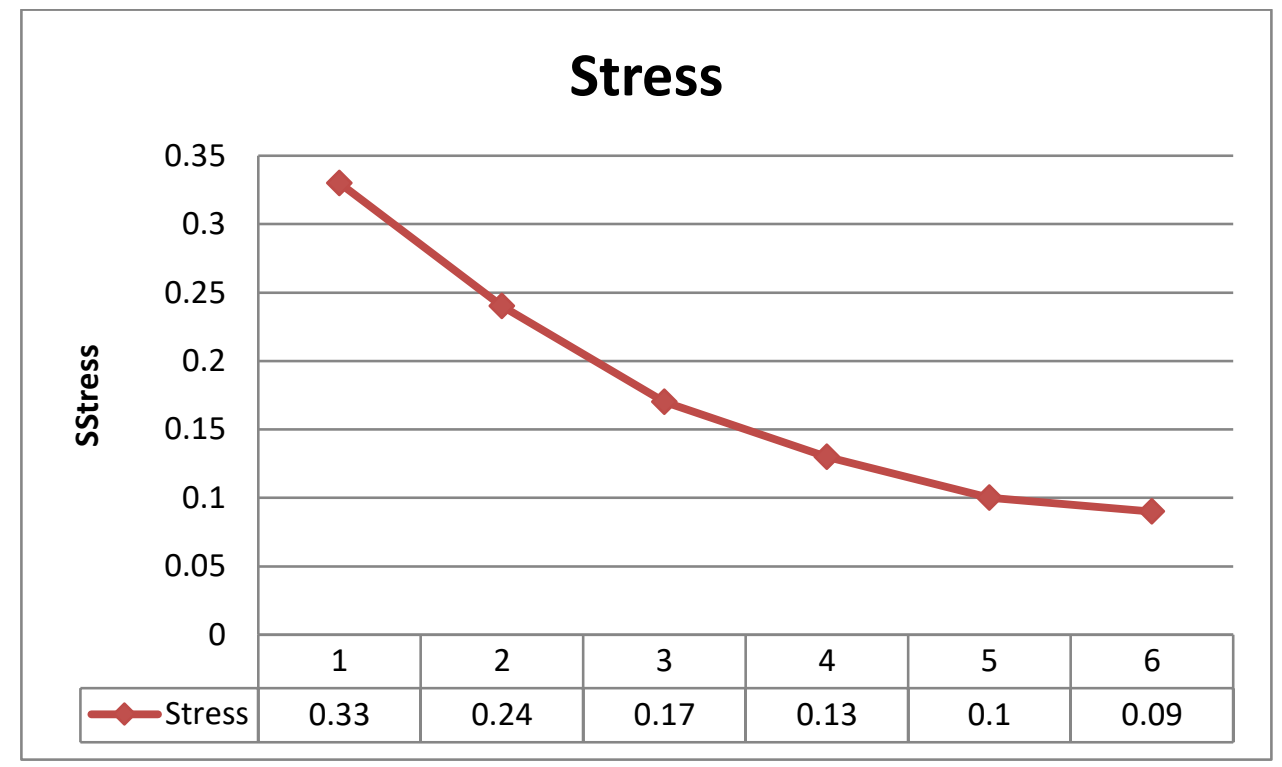

Figure 2: SStress values at different dimensions of TOEFL writing prompts.

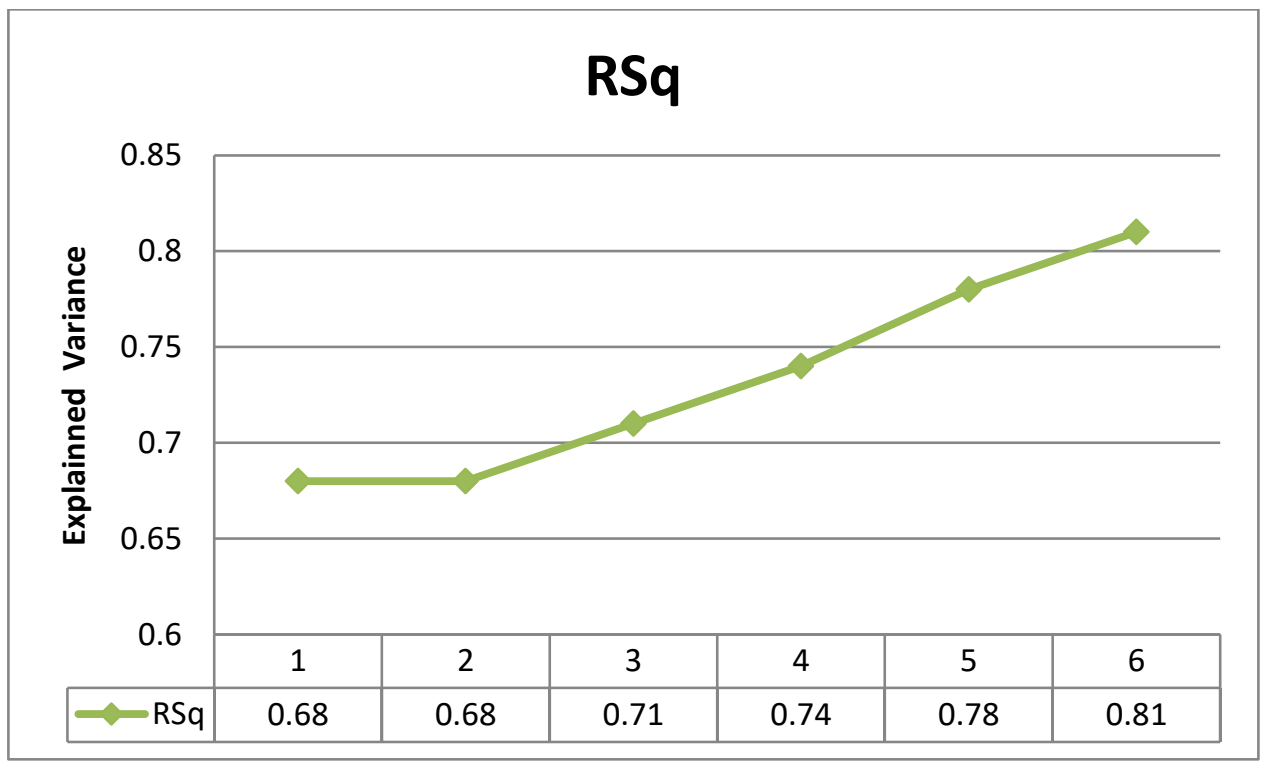

Figure 3: RSq values at different dimensions of TOEFL writing prompts. 
Figure 4 shows the results of the analysis using the PROXSCAL algorithm in the form of a scree plot.

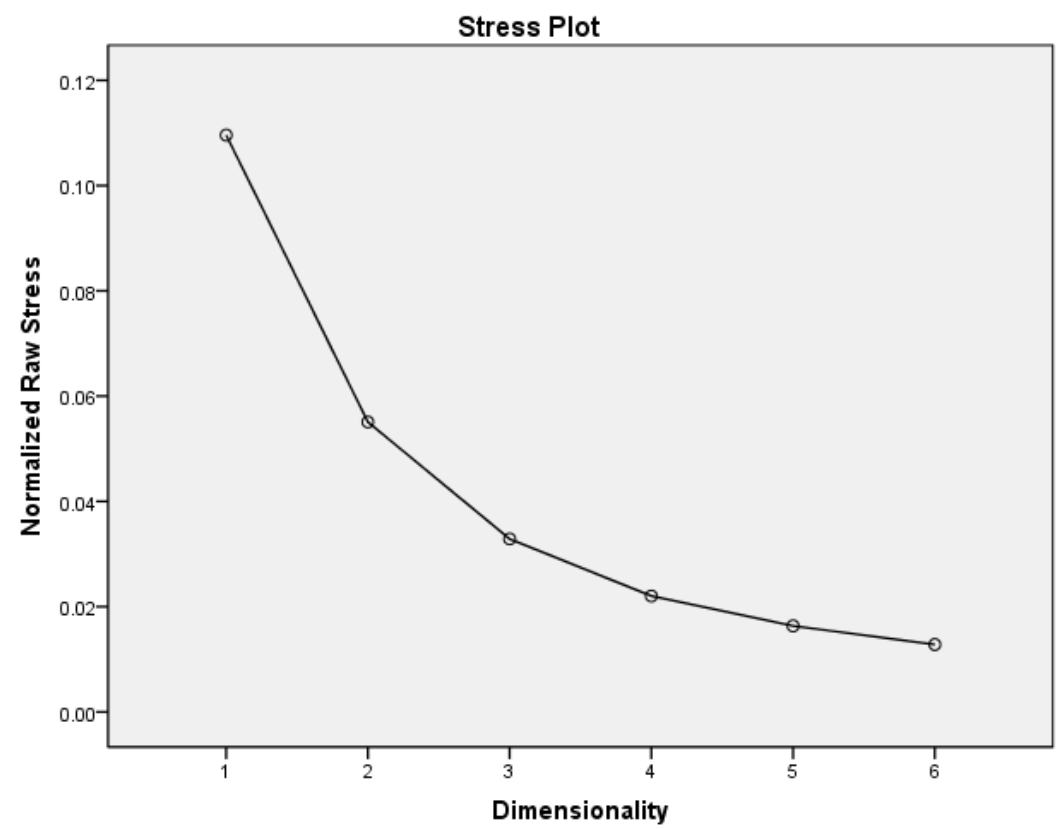

Figure 4: PROXSCAL stress plot for TOEFL writing prompts dimensions.

As the stress plot suggests, two to three dimensions are likely in the structure of TOEFL writing prompts.

\section{IELTS Prompts}

In this section, the ALSCAL and PROXSCAL results are presented for IELTS writing topics. Table 2 shows the results of ALSCAL analysis on the ordinal data of the IELTS writing prompts. The common indicators for goodness-of-fit are the loss function S-STRESS and the R squared (RSQ). The lower the S-STRESS is, the better the solution. For RSQ, we're seeking higher values. 
Table 2: SSTRESS and RSQ values for IELTS writing prompts from ALSCAL analysis.

\begin{tabular}{|l|l|l|l|l|l|l|l|l|}
\hline \multicolumn{9}{|c|}{ TOEFL Writing Prompts } \\
\hline \multirow{2}{*}{ Dim. } & \multicolumn{2}{|c|}{ Averaged } & \multicolumn{2}{c|}{ Matrix 1 } & \multicolumn{2}{c|}{ Matrix 2 } & \multicolumn{2}{c|}{ Matrix 3 } \\
\cline { 2 - 9 } & Stress & RSq & Stress & RSq & Stress & RSq & Stress & RSq \\
\hline 6 & .11 & .65 & .12 & .50 & .06 & .88 & .19 & .58 \\
\hline 5 & .13 & .63 & .16 & .44 & .08 & .86 & .13 & .59 \\
\hline 4 & .16 & .58 & .20 & .38 & .10 & .83 & .17 & .51 \\
\hline 3 & .21 & .56 & .25 & .35 & .13 & .80 & .21 & .52 \\
\hline 2 & .29 & .51 & .36 & .22 & .18 & .81 & .30 & .50 \\
\hline 1 & .39 & .55 & .50 & .26 & .26 & .81 & .38 & .58 \\
\hline
\end{tabular}

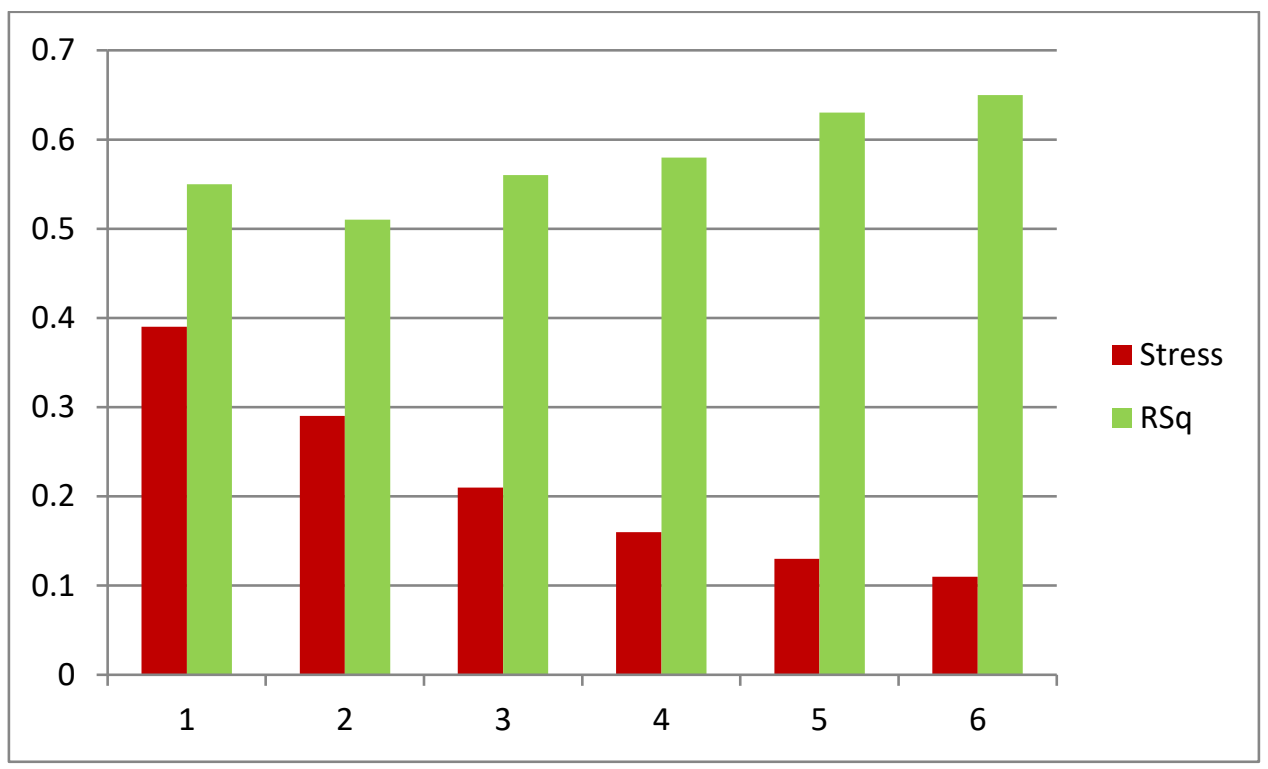

Figure 5: SStress and RSq values at different dimensions of IELTS Writing Prompts.

As the loss function values show, the STRESS improves noticeably going from two dimensions to three dimensions, after which the dip is gradual. The increase in RSQ is almost 
equal from one dimension to the next higher, except for the second to the third dimension, where a higher jump is noticed. Therefore, the ALSCAL results suggest a three-dimensional structure to the writing prompts of IELTS test.

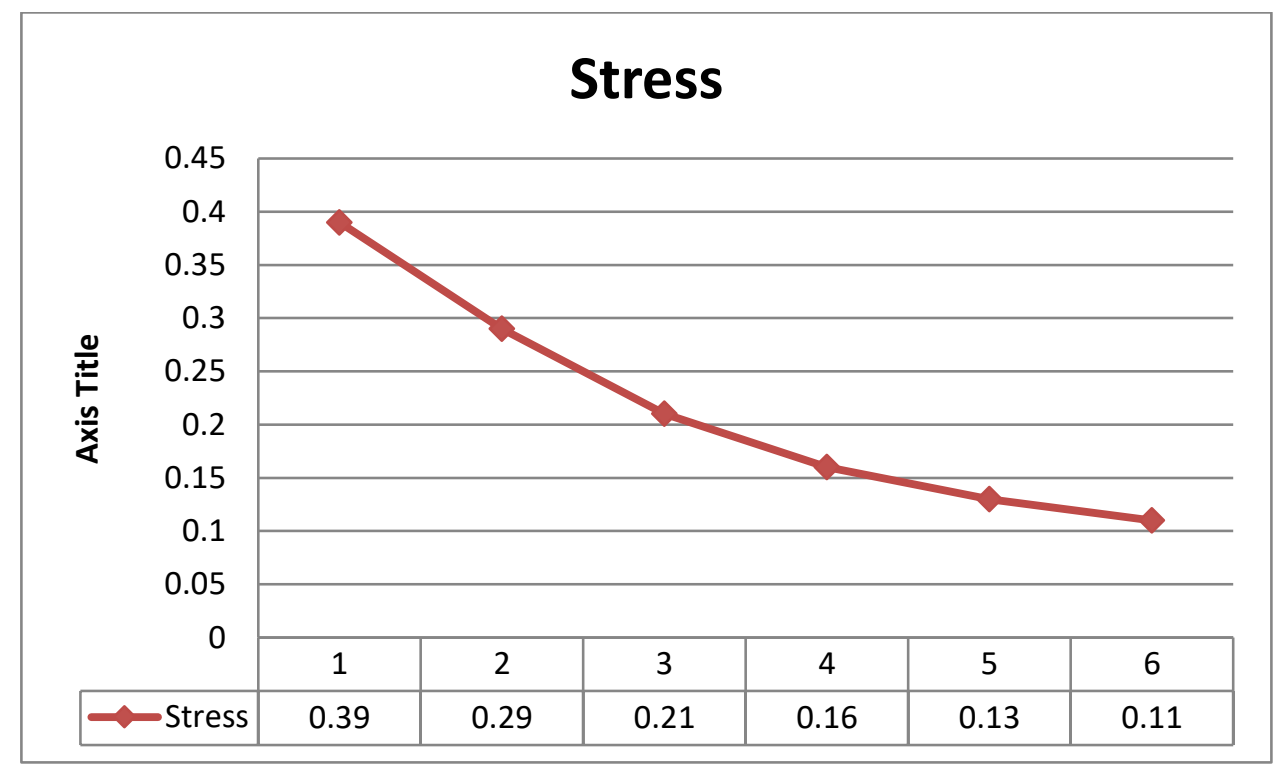

Figure 6: SStress values at different dimensions of IELTS writing prompts. 


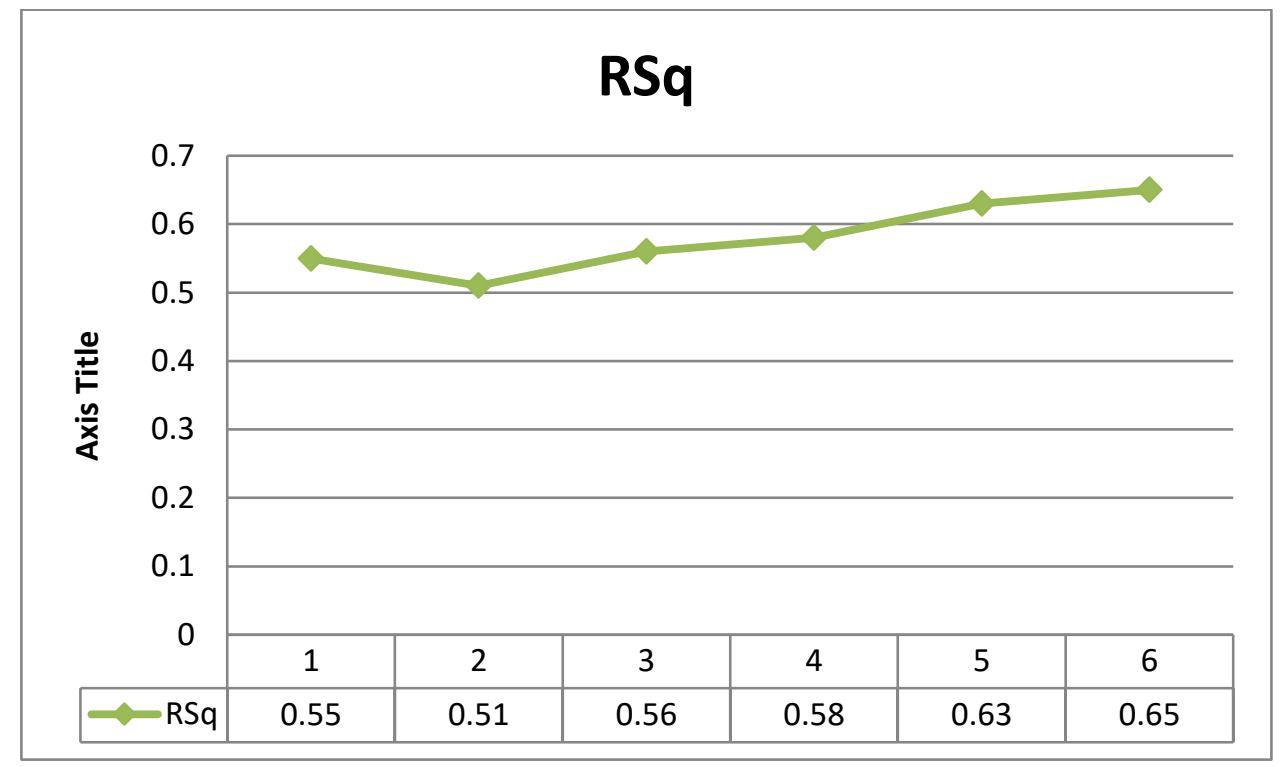

Figure 7: RSq values at different dimensions of IELTS writing prompts.

Figure 8 shows the results of the analysis using the PROXSCAL algorithm in the form of a scree plot.

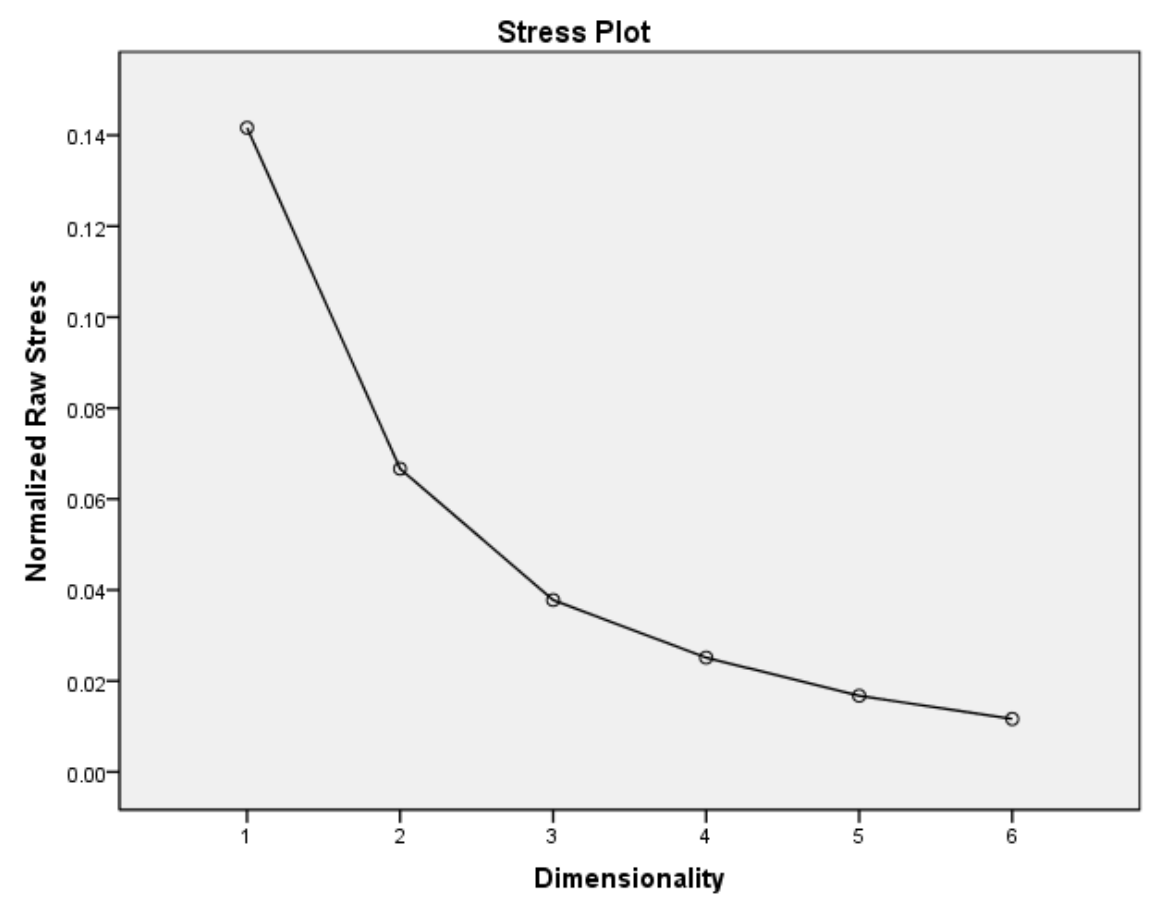

Figure 8: PROXSCAL scree plot for IELTS writing prompts dimensions. 
The PROXSCAL scree plot shows a two- or a three-dimensional structure to the IELTS writing prompts.

\section{Interpretation}

Davison and Sireci (2000, p. 335) suggest three criteria in determining and interpreting the dimensionality structure of MDS analysis results: model-data fit, interpretability of the solution and reproducibility (replication). The latter two go hand-in-hand. The model-data fit is judged using the STRESS (PROXSCAL) or S-STRESS (ALSCAL) values (the lower and closer to zero, the better fit), which indicates the difference between the scaled distances and transformed proximities (Davison \& Sireci, 2000). RSQ is another index which shows the proportion variance in the distances accounted for by the transformed data. For the sake of parsimony, the researcher needs to determine the lowest dimensionality which fully represents all aspects of the data structure (Ibid). In addition to the visual inspection of the dimensions, Kruskal and Wish (1978) also recommend interpreting the neighborhoods formed along the dimensions, especially when MDS gives a high-dimensional solution.

\section{Interpreting TOEFL Writing Prompts Dimensionality}

As both ALSCAL and PROXSCAL procedures showed, a two-dimensional interpretation is possible considering the SSTRESS value and the RSQ and the interpretability of the solution. Figure 9 shows the two-dimensional structure for the TOEFL writing prompts. The axes are rotated for better interpretation of the dimensions. 


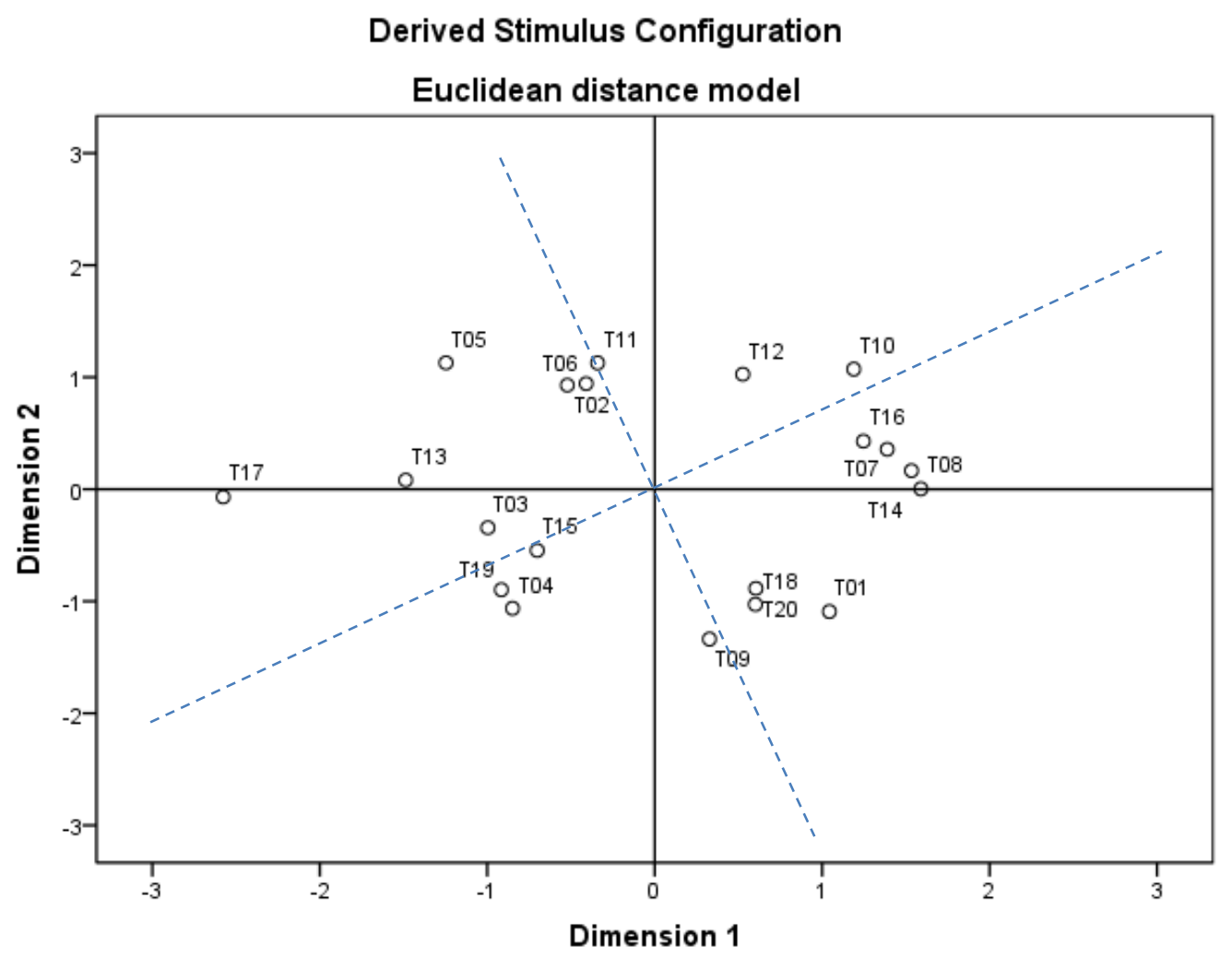

Figure 9: Two-dimensional structure for TOEFL writing prompts.

If we rotate the axis counterclockwise about 23 degrees, we can see that two coordinates of items are formed: T03, T04, T15, T19, T13; T10, T12, T16, T07, T08 and T14 on one dimension, and T02, T06, T11, T05; T09, T01, T18 and T20, on the other dimension. Now we can look at these prompts and see what characteristic(s) distinguishes them. What I see is that in the first group of prompts, the examinee is required to write about something personal and known through personal experience or affecting their "self". The second cluster of this dimension asks examinees to write about something distal from their personal experience; it is about society, environment or experiences to be gained in the future (e.g. running an owned business). I call this dimension Personal and Societal Development. The second dimension is trickier to interpret. Nevertheless, it can be seen that most prompts on this dimension pivot around 
evaluating the structures or components of a modern society in terms of their utility and justification, such as attending classes, the use of a zoo and spending money on space projects.

\section{Interpreting IELTS Writing Prompts Dimensionality}

As both ALSCAL and PROXSCAL procedures showed, a three-dimensional interpretation is possible considering the STRESS value and the RSQ and the interpretability of the solution. Figures 10 and 11 show the three-dimensional structure for the IELTS writing prompts.

\section{IELTS Prompts}

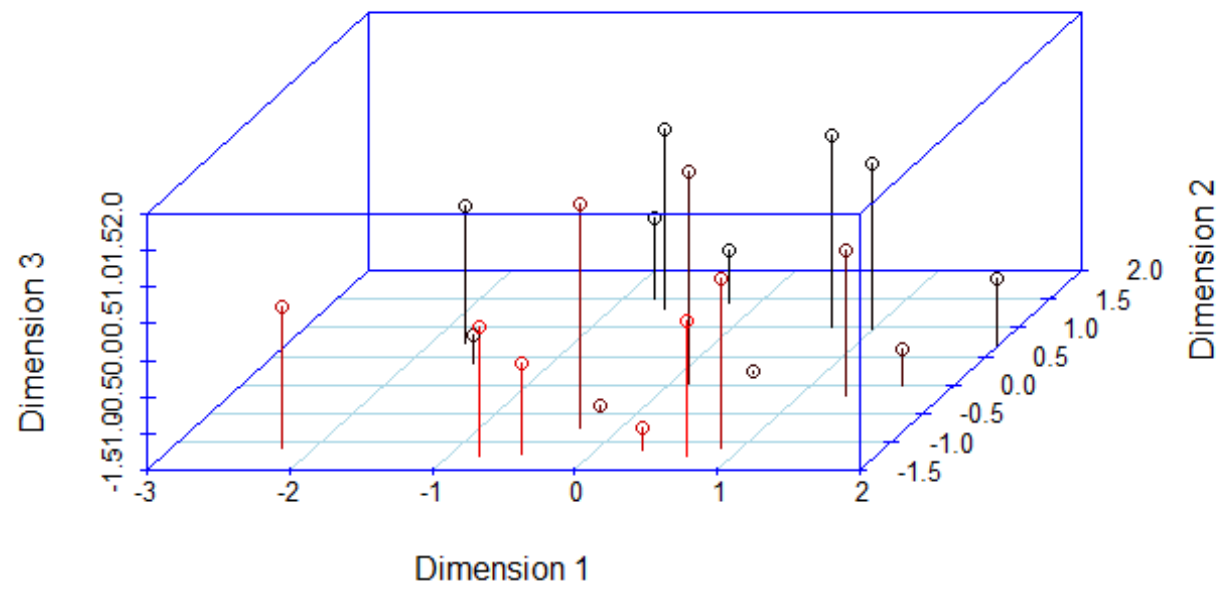

Figure 10: Three-dimensional structure for IELTS writing prompts. 


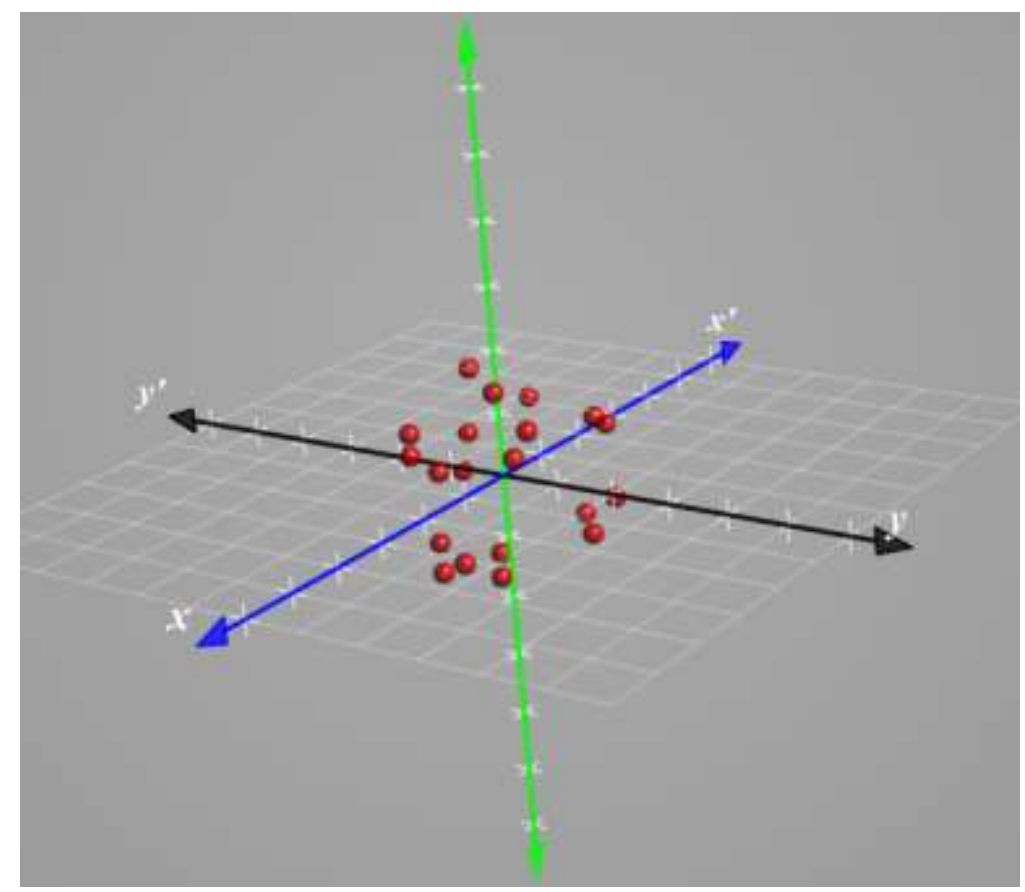

Figure 11: Three-dimensional structure for IELTS writing prompts.

The best way to identify the dimensions in $3 \mathrm{D}$ structure is to rotate the graph in different angles and perspectives. In doing so, prompts 1, 2, 4, 6, 7, 14 and 17 fall one dimension; prompts (2), (5), 9, 10, 11, 12, 13, (17), and 18 fall on the second dimension; and prompts 3, 5, 8, (14), 15, 16, 19, and 20 fall on the third dimension (prompts in parentheses are gravitated to more than one dimension). What I discern in the first set of prompts in the IELTS test is the dominance topics related to individual and their interaction with another individual or the society at large, such as motherhood, community service and children growing into social adults. The second dimension mostly includes topics about analyzing the issues of the society, such as salary disparity, effect of technology on relationships and health issues. The third dimension also threads social issues together, but regarding issues caused by social structure and not by social behaviors, such as censorship, capital punishment and international relationships. The second and third dimensions are similar to each other and probably a single dimension. 


\section{Discussion}

To what extent test items measure the construct or the content is a matter of test validation and score interpretation. An item must be clear of irrelevant constructs or factors for the score interpretation to be valid. This study differs from a typical test validation study in that the items (writing prompts) in this study never appear together as a test. Nevertheless, they do belong to the same testing purpose: assessing the writing proficiency of non-English speaking candidates. Thus performing a validation study on the prompts to find different dimensions or categories of items is justified. Finding different dimensions or categories of prompts helps comparability of test scores and the appropriateness of the prompts for different examinees groups, in terms of education, age and cognitive attributes.

In both the TOEFL and IELTS writing prompts, one category items asks examinees to write about personal and social experiences, which are more immediate and concrete to them. However, in a second category, the examinees in both tests are required to think more abstractly as problem solvers or judges regarding the social issues and problems. The IELTS prompts add the complexity by projecting a third dimension of detached factors affecting the social structure, which is more abstract and requires higher cognitive ability to resolve. These issues may pose preferential selection of prompts for different examinees with different education, age and cognitive attributes.

\section{Implications of the Study}

The results of the present study reflect implications for test developers, test takers and test score users. For test developers, the results show that writing prompts need to be aligned with different attributes of audiences or more and exhaustive choices be given to the test takers 
in selecting the prompts. The test takers are reminded that writing proficiency is not just defined by knowing about the language, but rather it involves different levels of thinking and prior knowledge and experiences. For test users, the implications regard determining cut-off scores for different tests and score comparability across different writing tests.

\section{Limitations of the Study}

I think the following limitations are noteworthy in generalizing the results of this study:

1. Only three raters were recruited. More raters will produce more reliable measures.

2. The study comprises 780 pairs of prompts. This is very lengthy and can cause systematic error.

3. The raters were language teachers. So it is probable that they were familiar with the writing prompts.

4. Extreme response styles were detected in two of the raters but not in one. Extreme responses introduce measurement imprecision (through affecting the mean in an extreme way).

\section{Suggestions for Further Research}

The future researchers are encouraged to replicate this study with limitations ameliorated and adding external variables such as test scores for further verification of the dimensionality of the prompts. Also, conducting a similar study on speaking prompts would be interesting. 


\section{References}

American Educational Research Association (AERA), American Psychological Association (APA) \& National Council on Measurement in Education (1999). Standards for educational and psychological testing. Washington, D.C: AERA.

Borg, I. Groenen, P. J. F. (2005). Modern multidimensional scaling. 2nd ed. New York: Springer.

Borg, I. Groenen, P. J. F., Mair, P. (2013). Applied multidimensional scaling. New York: Springer.

Chapelle, C. A, Enright, M. K. \& Jamieson, J. (2010). Does and argument-based approach to validity make a difference? Educational Measurement: Issues and Practice, Vol. 29, 1.

Cox, T. F., \& Cox, M. A. A. (2001). Multidimensional scaling. 2nd ed. USA: Chapman and Hall/CRC

Davison, M. \& Sireci, S. G. (2000). Multidimensional scaling. In Tinsley, H. E. A \& Brown, S. D. (2000), Handbook of applied multivariate statistics and mathematical modeling. New York: Academic Press.

Kruskal, J. B. \&Wish, M. (1978). Multidimensional scaling. California: Sage Publications.

Sireci, S. G. \& Colvin, K. F. (2013). Scientific Knowledge for Teaching Item Review Summary Report. Center for Educational Assessment Research Report No. 843. Amherst, MA: Center for Educational Assessment, University of Massachusetts.

Sireci, S. G. \& Geisinger, K. F. (1995). Using subject-matter experts to assess content representation: an MDS analysis. Applied Psychological Assessment, Vol. 19, No. 3.

Spence, I. \& Domoney, D. W. (1974). Single subject incomplete designs for nonmetric multidimensional scaling. Psychometrica, 39, 469-490. 